\title{
BUILDING RELIABLE MODELS OF M DWARF CHROMOSPHERES: THE SPECTRAL DIAGNOSTICS
}

\author{
Pablo J. D. Mauas ${ }^{1}$ \\ Instituto de Astronomía y Física del Espacio (IAFE), Casilla de Correo 67, Sucursal 28, 1428 Buenos Aires, Argentina; pablo@iafe.uba.ar \\ Received 2000 January 3; accepted 2000 March 21
}

\begin{abstract}
As part of an ongoing project to study how reliable are the chromospheric models constructed semiempirically to fit the chromospheric spectrum of cold stars, in this paper we study how changes in the assumed chromospheric structure of $\mathrm{dM}$ and $\mathrm{dMe}$ stars affect the emitted spectrum. In this way, we study if a given set of features can wholly determine the atmospheric structure, how large are the indeterminations in the atmospheric parameters deduced, and to what extent the atmospheric model computed can be considered unique. We find that the profiles of the $\mathrm{Ca}$ II $\mathrm{K}$ line or the $\mathrm{Na} \mathrm{D}$ lines can be used to determine the structure of the $T_{\min }$ region and that the $\mathrm{H} \alpha$ profile can provide good information on the structure of the chromosphere. The Ly $\alpha$ flux, in turn, can be used to constrain the position of the transition region.

Subject headings: stars: atmospheres — stars: chromospheres - stars: late-type
\end{abstract}

\section{INTRODUCTION}

Semiempirical atmospheric models have been constructed for a number of cool stars to explain the differences in the observed spectra (Cram \& Mullan 1979; Giampapa, Worden, \& Linsky 1982; Thatcher, Robinson, \& Rees 1991; Houdebine \& Doyle 1994b). By "semiempirical” we mean that, given a $T$ versus $z$ distribution, the non-LTE populations for hydrogen, and perhaps other species, are computed, solving simultaneously the equations of hydrostatic equilibrium, radiative transfer, and statistical equilibrium.

Once the calculations are completed for a particular atmospheric model, the emerging profiles for a given set of lines are computed, and the results are compared with the observations. Then the assumed $T$ versus $z$ distribution is modified until a satisfactory match between the observations and the prediction of the calculations is obtained.

For example, in a series of papers (Mauas \& Falchi 1994, 1996; Mauas et al. 1997, hereafter Papers I, II, and III, respectively) we built a series of atmospheric models for some $\mathrm{dM}$ stars in the quiescent state and for a big flare on the dMe star AD Leo.

However, there are several intrinsic problems regarding this approach. The most important lies in the uniqueness of the models computed in this way. In other words, knowing that a particular atmosphere would emit a line profile like the one we observe for a given star does not imply that the star has indeed this atmospheric structure, since we do not know whether some other atmosphere would produce the same profile.

This problem is, of course, larger when the modeling is based only on matching a small number of spectral features. For example, the indetermination is larger when the only thing to be matched is the $\mathrm{H} \alpha$ profile than when the $\mathrm{Ca}$ II $\mathrm{K}$ line is also used (let alone when only line fluxes, or even Balmer decrements, are used, instead of the whole line profile).

Related to this problem is the question of which are the diagnostic tools needed to constrain the atmosphere. Normally, one would try to model as large a number of spectral features as possible, but this is not always possible. There-

\footnotetext{
${ }^{1}$ Member of the Carrera del Investigador Científico, CONICET, Argentina.
}

fore, it is very important to know which profiles give redundant information and which observations are needed to constrain different regions of the atmosphere, particularly when planning the observations.

Another related problem is the one of the errors in the atmospheric models. How accurate is the temperature (for example, the minimum temperature) determined from a spectral feature? And how accurate is the position (for example, of the transition region) determined from a line flux?

As part of an ongoing project of constructing atmospheric models for cool stars, in Paper IV of this series (Falchi \& Mauas 1998) we began a study of how reliable the semiempirical models are. In that paper we studied the impact of the assumptions usually made to simplify the modeling process. We showed that ignoring line blanketing, assuming metals in LTE, or computing the Ly $\alpha$ and the $\mathrm{Ca}$ II $\mathrm{K}$ lines with complete redistribution (CRD) produces large differences in the computed line profiles and therefore in the model adopted for the star under study.

In this paper we study how different changes in the chromospheric models modify the different spectral features. In this way, we study if a given set of features can wholly determine the atmospheric structure, how large are the indeterminations in the atmospheric parameters deduced, and to what extent the atmospheric model computed can be considered unique. To do this, we slightly modify the atmospheric models obtained for the dM 3.5 star G1 588 and the dMe 3.5 star AD Leo (discussed in Papers III and I). We intend to study how the emergent spectrum changes when the temperature in different regions of the chromosphere is changed and therefore how reliably the atmosphere can be modeled.

In $\S 2$ we explain how the models are built. In $\S 3$ we discuss the different diagnostic tools used to model the atmospheres. Finally, in $\S 4$ we discuss the results.

\section{ATMOSPHERIC MODELS}

The modeling was done using the program Pandora developed, and kindly provided, by Dr. E. H. Avrett. For details on how the models are built, we refer the reader to Paper IV. Here we just point out that, for every $T$ versus $z$ distribution, we self-consistently compute non-LTE popu- 


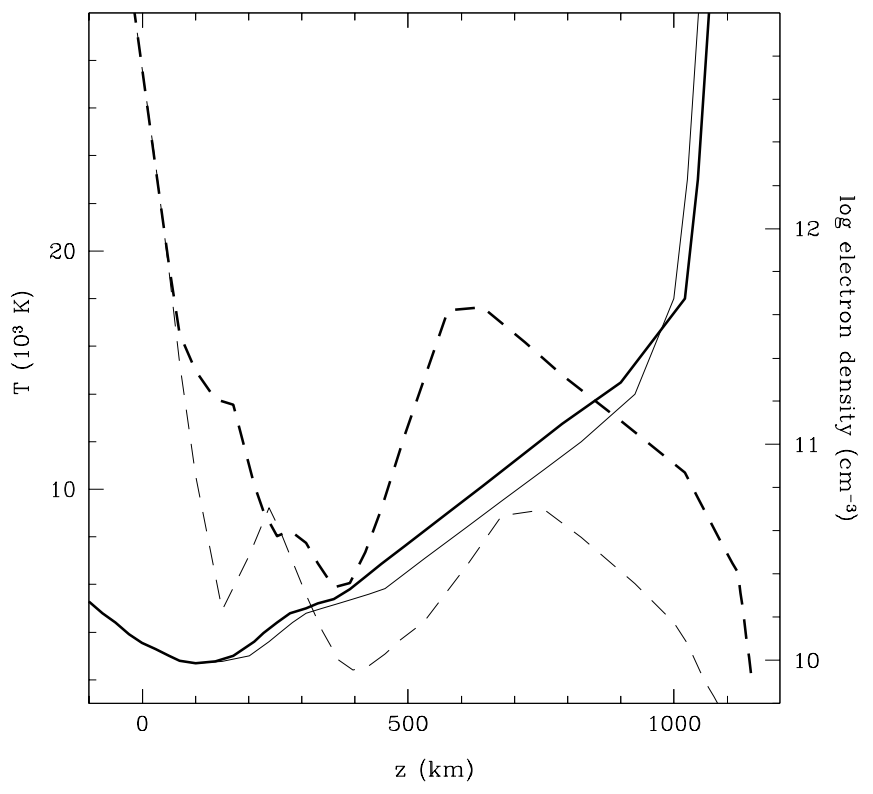

FIG. 1.-Temperature distribution (solid lines, left scale) and electron density (dashed lines, right scale) for our models. Thin lines: model C (Gl 588, see Paper III); thick lines: model H (Ad Leo, see Paper I).

lations for 15 levels of $\mathrm{H}$ (including all its 105 bound-bound transitions) and seven metallic species and that we include atomic and molecular line blanketing, using the compilation from Kurucz (1991).

We build two grids of atmospheric models, considering as the base model for each grid the models of the dM star Gl 588 (see Paper III) and the dMe star AD Leo (see Paper I). We refer the reader to these papers for a detailed discussion of the atmospheric structures. In Figure 1 we show the temperature and electron density distributions for both base models, which we will call our "cold" or C model, and our "hot" or H model, from now on.

Our atmospheric grids are not aimed to match the full range of observations available for $M$ dwarfs, as has been done, for example, by the Armagh group (see, for example, Andretta, Doyle, \& Byrne 1997). Since our goal is to under-

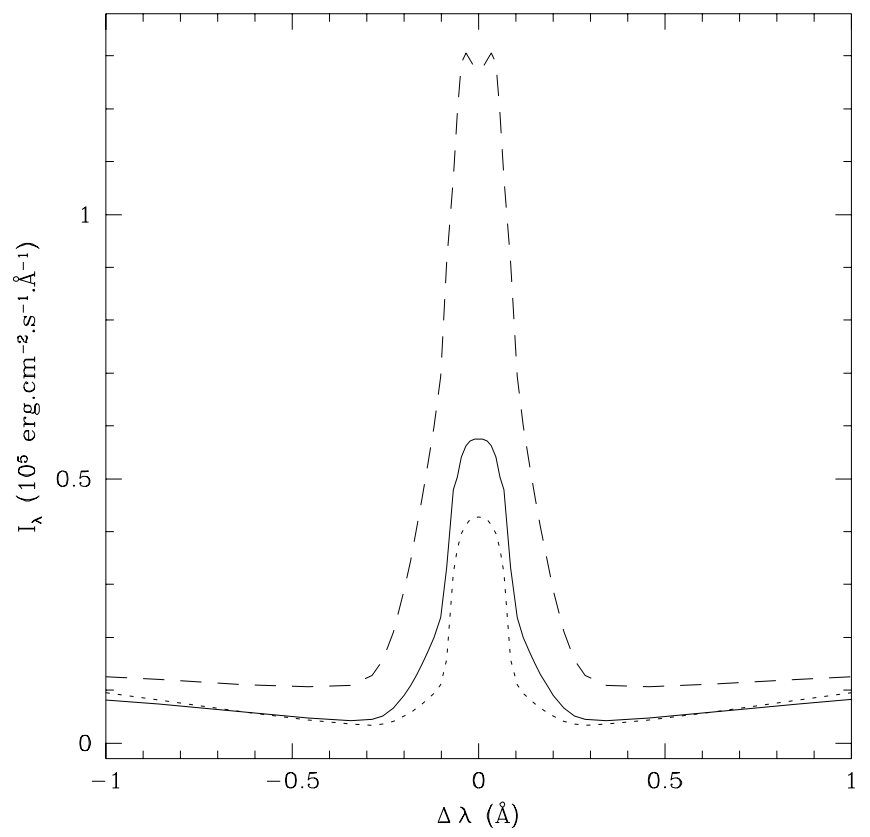

FIG. 2.-Profile of the Ca II K line for three models of our cold series. Solid line: model C; dashed line: model $\mathrm{CM}++$; dotted line: model $\mathrm{CR}+$.

stand how well we know the structure of a given star from the emitted spectrum, the changes in our models cover the range of atmospheres producing an output spectrum more or less similar to the one of our chosen star. Therefore, each grid is built modifying slightly the base model, to test how small changes in the structure of the atmosphere are reflected in the considered spectral features, i.e., the $\mathrm{Ca}$ II $\mathrm{K}$ line, the $\mathrm{Na} \mathrm{D}$ lines, the highest Balmer lines, and the Ly $\alpha$ line. However, we will see that these changes are large enough to produce large variations in the considered spectral features (e.g., see Fig. 2 below). The main characteristics of the grid models are included in Table 1, for our cold series, and in Table 2, for our hot series.

Since we are interested in the chromospheric properties, we left unchanged the photospheric structure, and we separately modified $(a)$ the minimum temperature, shown in

TABLE 1

ATMOSPHeric PARAMETERS FOR OUR COLD MODELS

\begin{tabular}{|c|c|c|c|c|c|c|c|c|c|c|c|c|c|c|c|c|}
\hline $\begin{array}{l}\text { Name } \\
\text { (1) }\end{array}$ & $\begin{array}{l}T_{\min } \\
\text { (2) }\end{array}$ & $\begin{array}{c}z_{4400} \\
\text { (3) }\end{array}$ & $\begin{array}{c}T_{500} \\
(4)\end{array}$ & $\begin{array}{c}T_{825} \\
(5)\end{array}$ & $\begin{array}{c}z_{18,000} \\
\text { (6) }\end{array}$ & $\begin{array}{l}\mathrm{K} 1 \\
\text { (7) }\end{array}$ & $\begin{array}{l}\mathrm{K} 3 \\
(8)\end{array}$ & $\begin{array}{l}\mathrm{H} \alpha \\
(9)\end{array}$ & $\begin{array}{l}\mathrm{H} \gamma \\
(10)\end{array}$ & $\begin{array}{l}F_{\mathrm{H} \alpha} \\
(11)\end{array}$ & $\begin{array}{l}F_{\mathbf{H} \gamma} \\
(12)\end{array}$ & $\begin{array}{l}F_{\text {Ly } \alpha} \\
(13)\end{array}$ & $\begin{array}{c}W_{\mathrm{eq}}(\mathrm{H} \alpha) \\
\text { (14) }\end{array}$ & $\begin{array}{c}W_{\mathrm{eq}}(\mathrm{H} \gamma) \\
(15)\end{array}$ & $\begin{array}{c}\mathrm{H} \alpha / \mathrm{H} \gamma \\
(16)\end{array}$ & $\begin{array}{c}F_{\mathrm{Ly} \alpha} / F_{\mathrm{H} \alpha} \\
\quad(17)\end{array}$ \\
\hline $\mathrm{C} \ldots \ldots \ldots \ldots \ldots$ & 2700 & 283 & 6564 & 12000 & 1000 & 0.42 & 5.1 & 11.9 & 4.7 & -1.5 & -0.033 & 2.9 & 0.51 & 0.057 & 2.5 & -1.9 \\
\hline $\mathrm{CM}+\ldots \ldots \ldots$ & 2800 & & & & & 0.66 & 7.7 & 13.6 & 5.7 & -1.2 & 0.010 & 3.7 & 0.38 & -0.017 & 2.4 & -3.0 \\
\hline $\mathrm{CM}++\ldots \ldots$ & 2900 & & & & & 1.1 & 11.8 & 15.9 & 6.7 & -0.67 & 0.075 & 4.4 & 0.22 & -0.13 & 2.4 & -6.6 \\
\hline $\mathrm{CM}-\ldots \ldots \ldots$ & 2600 & & & & & 0.27 & 3.4 & 10.7 & 4.2 & -1.8 & -0.058 & 2.6 & 0.60 & 0.10 & 2.5 & -1.4 \\
\hline $\mathrm{CR}+\ldots \ldots \ldots$ & & 307 & & & & 0.33 & 3.4 & 11.0 & 4.3 & -1.7 & -0.052 & 2.7 & 0.56 & 0.090 & 2.5 & -1.6 \\
\hline $\mathrm{CR}-\ldots \ldots \ldots$ & & 259 & & & & 0.65 & 8.2 & 12.9 & 5.3 & -1.3 & -0.064 & 3.3 & 0.43 & 0.110 & 2.4 & -2.5 \\
\hline $\mathrm{CC}+\ldots \ldots \ldots$ & & & 6964 & 12400 & & 0.42 & 5.7 & 12.3 & 5.1 & -1.5 & -0.019 & 3.2 & 0.50 & 0.033 & 2.4 & -2.2 \\
\hline $\mathrm{CC}-\ldots \ldots \ldots$ & & & 6164 & 11600 & & 0.42 & 4.4 & 11.3 & 4.4 & -1.6 & -0.047 & 2.7 & 0.52 & 0.082 & 2.6 & -1.7 \\
\hline $\mathrm{CS}+\ldots \ldots \ldots$ & & & & 12800 & & 0.42 & 5.5 & 12.3 & 5.0 & -1.5 & -0.022 & 3.2 & 0.50 & 0.038 & 2.4 & -2.1 \\
\hline $\mathrm{CS}-\ldots \ldots \ldots$ & & & & 11200 & & 0.42 & 4.7 & 11.2 & 4.4 & -1.6 & -0.047 & 2.8 & 0.53 & 0.083 & 2.5 & -1.7 \\
\hline $\mathrm{CZ}+\ldots \ldots \ldots$ & & & & & 1040 & 0.42 & 6.0 & 12.1 & 4.8 & -1.5 & -0.030 & 2.6 & 0.50 & 0.053 & 2.5 & -1.7 \\
\hline $\mathrm{CZ}-\ldots \ldots \ldots$ & & & & & 960 & 0.42 & 5.1 & 11.5 & 4.7 & -1.5 & -0.034 & 3.6 & 0.50 & 0.060 & 2.4 & -2.3 \\
\hline
\end{tabular}

NoTE.-Parameters include the minimum temperature $T_{\min }$; the temperatures at 500 and $825 \mathrm{~km}$ in the chromosphere, $T_{500}$ and $T_{825}$; and the heights at which the temperature is $4400 \mathrm{~K}$ and $18,000 \mathrm{~K}, z_{4400}$ and $z_{18,000}$ (these two heights refer to the positions of the first chromospheric rise and of the transition region). Blank entries mean that the value is the same as the one for the base model, given in the first row. For each model we give the Ca II K1 minimum; the maximum intensity $\mathrm{K} 3$ convolved with a spectral resolution of $0.15 \AA$; the $\mathrm{H} \alpha$ and $\mathrm{H} \gamma$ central intensities in $10^{4} \mathrm{ergs} \mathrm{cm}^{-2} \mathrm{~s}^{-1} \AA^{-1}$; the line fluxes with the continuum subtracted for $\mathrm{H} \alpha, \mathrm{H} \gamma$, and Ly $\alpha$ in $10^{5} \mathrm{ergs} \mathrm{cm}^{-2} \mathrm{~s}^{-1}$; the equivalent width for $\mathrm{H} \alpha$ and $\mathrm{H} \gamma$ in $\AA$; and some ratios of the above parameters. 
TABLE 2

Atmospheric Parameters for OUR Hot Models

\begin{tabular}{|c|c|c|c|c|c|c|c|c|c|c|c|c|c|}
\hline $\begin{array}{c}\text { Name } \\
\text { (1) }\end{array}$ & $\begin{array}{c}T_{\min } \\
(2)\end{array}$ & $\begin{array}{c}z_{4400} \\
\text { (3) }\end{array}$ & $\begin{array}{c}T_{400} \\
\text { (4) }\end{array}$ & $\begin{array}{c}T_{900} \\
(5)\end{array}$ & $\begin{array}{c}z_{18,000} \\
\text { (6) }\end{array}$ & $\begin{array}{c}D_{\min } \\
(7)\end{array}$ & $\begin{array}{l}\mathrm{H} \alpha \\
(8)\end{array}$ & $\begin{array}{l}\mathrm{H} \gamma \\
(9)\end{array}$ & $\begin{array}{l}F_{\mathbf{H} \alpha} \\
(10)\end{array}$ & $\begin{array}{l}F_{\text {Ly } \alpha} \\
(11)\end{array}$ & $\begin{array}{c}F_{\mathrm{Mg} \mathrm{II} k} k \\
(12)\end{array}$ & $\begin{array}{c}\mathrm{H} \alpha / \mathrm{H} \gamma \\
\text { (13) }\end{array}$ & $\begin{array}{c}F_{\mathrm{Ly} \alpha} / F_{\mathrm{H} \alpha} \\
(14)\end{array}$ \\
\hline $\mathrm{H} \ldots \ldots \ldots \ldots$ & 2700 & 253 & 5990 & 14470 & 1020 & 0.54 & 17.2 & 5.0 & 13.8 & 21.4 & 13.9 & 3.4 & 1.6 \\
\hline $\mathrm{HM}+\ldots \ldots$ & 2620 & & & & & 0.80 & 20.0 & 6.3 & 16.1 & 23.7 & 18.4 & 3.2 & 1.5 \\
\hline $\mathrm{HM}-\ldots .$. & 2780 & & & & & 0.46 & 15.1 & 4.2 & 11.8 & 20.2 & 10.8 & 3.6 & 1.7 \\
\hline $\mathrm{HR}+\ldots .$. & & 271 & & & & 0.44 & 15.0 & 4.2 & 10.9 & 19.4 & 16.5 & 3.6 & 1.8 \\
\hline $\mathrm{HR}-\ldots .$. & & 235 & & & & 0.71 & 20.5 & 6.9 & 17.0 & 31.6 & 11.1 & 3.0 & 1.9 \\
\hline $\mathrm{HC}+\ldots \ldots$ & & & 6240 & 14720 & & 0.54 & 17.6 & 5.2 & 14.0 & 22.1 & 16.5 & 3.4 & 1.6 \\
\hline $\mathrm{HC}-\ldots .$. & & & 5740 & 14220 & & 0.54 & 16.8 & 4.8 & 13.5 & 20.8 & 11.5 & 3.5 & 1.6 \\
\hline $\mathrm{HS}+\ldots \ldots$. & & & & 15070 & & 0.54 & 17.2 & 5.2 & 13.9 & 22.2 & 9.7 & 3.3 & 1.6 \\
\hline $\mathrm{HS}-\ldots \ldots$ & & & & 13870 & & 0.54 & 17.3 & 4.8 & 13.9 & 21.2 & 19.8 & 3.7 & 1.5 \\
\hline $\mathrm{HZ}+\ldots \ldots$ & & & & & 1120 & 0.54 & 16.9 & 5.0 & 13.6 & 19.4 & 13.9 & 3.4 & 1.4 \\
\hline $\mathrm{HZ}-\ldots .$. & & & & & 920 & 0.54 & 17.6 & 5.0 & 14.0 & 28.9 & 13.9 & 3.5 & 2.1 \\
\hline
\end{tabular}

NoTE.-Parameters include the minimum temperature $T_{\min }$; the temperatures at 400 and $900 \mathrm{~km}$ in the chromosphere, $T_{400}$ and $T_{900}$; and the heights at which the temperature is $4400 \mathrm{~K}$ and $18,000 \mathrm{~K}, z_{4400}$ and $z_{18,000}$ (these two heights refer to the positions of the first chromospheric rise and of the transition region). For each model we give the Na D2 minimum $\mathrm{D}_{\min }$; the $\mathrm{H} \alpha$ and $\mathrm{H} \gamma$ maximum intensities in $10^{5}$ ergs cm ${ }^{-2} \mathrm{~s}^{-1} \AA^{-1}$; the line fluxes with the continuum subtracted for $\mathrm{H} \alpha$, Ly $\alpha$, and the Mg II $k$ line in $10^{5}$ ergs $\mathrm{cm}^{-2} \mathrm{~s}^{-1}$; and some ratios of the above parameters.

column (2) of the tables (models $\mathrm{M}+, \mathrm{M}++$, and $\mathrm{M}-$ ); $(b)$ the depth of the first chromospheric rise, determined by the height at which $T=4400 \mathrm{~K}$, given in column (3) of the tables (models R + and R-); (c) the temperature of the high chromosphere, shown for two depths in columns (4) and (5) of the tables (models $\mathrm{C}+$ and $\mathrm{C}-$ ); $(d)$ the chromospheric slope, also determined by the temperatures shown in columns (4) and (5) (models $\mathrm{S}+$ and $\mathrm{S}-$ ); and (e) the position of the transition region, indicated by the height at which $T=18,000 \mathrm{~K}$, shown in column (6) (models $\mathrm{Z}+$ and $\mathrm{Z}-$ ).

In Tables 1 and 2 we also give, for each model, the intensity and the flux at the stellar surface of the features we used to build and test our models. We also give some ratios between different features to help analyze the results.

\section{THE SPECTRAL DIAGNOSTIC}

\subsection{The $\mathrm{Ca}$ II $\mathrm{K}$ Line}

For years, the $\mathrm{K} 1$ minimum of the $\mathrm{Ca}$ II $\mathrm{K}$ line has been used as a diagnostic of the structure of the temperature minimum in the Sun and other cool stars (e.g., Ayres \& Linsky 1976; Avrett 1985). In Figure 2 we show the computed profiles of the $\mathrm{K}$ line for three models of our cold series. The difference in the $\mathrm{K} 1$ minimum can indeed be noticed.

In Figure 3 we show the source function for the $\mathrm{K}$ line for the three models included in Figure 2. We see that the K1 minimum is indeed formed at the temperature minimum, and that the source function at this depth is strongly coupled to the Planck function. This fact makes much easier the interpretation of the observations of K1. As a consequence of this coupling, the intensity in the $\mathrm{K} 1$ minimum changes with the minimum temperature $T_{\min }$ and with the position of the first chromospheric rise $z_{4400}$ but remains unchanged when the temperatures of the high and midchromosphere are modified, as can be seen in Table 1.

Therefore, the value of the $\mathrm{K} 1 \mathrm{minimum}$ is a very useful tool to build the atmospheric models in the minimum temperature region, since the temperature and width of the $T_{\min }$ region can be adjusted first, and the other spectral signatures adjusted afterwards, modifying only the midand high chromosphere. Furthermore, since most of the calcium in the stellar atmosphere is in the form of $\mathrm{Ca}$ II, computing the line profiles is rather straightforward and insensitive to any assumptions on the ionization balance, something that can affect the line profiles of minority ions like, for example, the $\mathrm{Ca}$ I line at $4227 \AA$.

We note that variations of the $T_{\min }$ of $100 \mathrm{~K}$ give a relative variation of the K1 of around $50 \%$. Since the precision of the spectrophotometric observations is usually better than $50 \%$, we can establish the $T_{\min }$ value with a precision higher than $100 \mathrm{~K}$. On the other hand, since for these cool stars the emission in the blue part of the spectrum is low, and this is particularly true for $\mathrm{K} 1$, long integration times are needed to obtain values of this feature with a good signal-to-noise ratio.

In Table 1 it can be seen that models $\mathrm{CM}+$ and $\mathrm{CR}-$ have the same $\mathrm{K} 1$ intensity, meaning that this feature can be

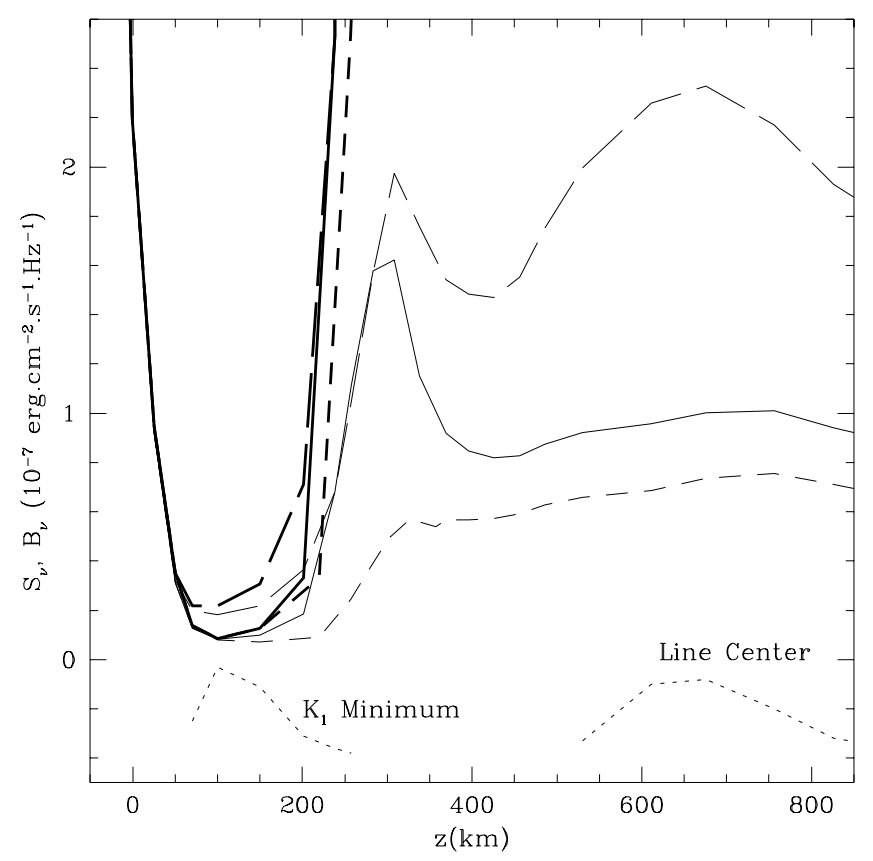

FIG. 3.- Source function (thin lines) and Planck function (thick lines) for the Ca II K line for three models of our cold series. Solid lines: model C; short-dashed lines: model $\mathrm{CM}++$; long-dashed lines: model $\mathrm{CR}+$. The dotted lines in the lower part of the figure are the contribution functions for the $\mathrm{K} 1 \mathrm{minimum}$ and the line center $\mathrm{K} 3$ for model $\mathrm{C}$. 
increased either by increasing the temperature minimum or by starting the chromospheric rise at lower heights. In Figure 4 we show a detail of the K1 region of the profiles for these two models. It can be seen that, although the intensity of $\mathrm{K} 1$ is indeed the same for the two models, there is a difference in the width of the emission feature, formed just above $T_{\min }$. This difference can be used to further constrain the models in this region if observations with good resolution are available. Although the difference between these profiles can appear too subtle, it should be noted that it is caused by a displacement of the chromospheric rise of only $24 \mathrm{~km}$.

In this paper we compute the profiles of the $\mathrm{Ca}$ II $\mathrm{K}$ and $\mathrm{H}$ lines with the partial redistribution (PRD) treatment explained in Paper IV. In that paper we showed that if complete redistribution is assumed, the $\mathrm{K} 1$ minimum is twice as intense as the one computed in PRD. As the changes introduced by the CRD assumption are larger than the ones obtained by modifying the atmospheric models, it is important to compute the $\mathrm{K}$ and $\mathrm{H}$ lines in PRD if they are going to be used for this kind of modeling.

In principle, the central intensity of the $\mathrm{K}$ line, $\mathrm{K} 3$, can be used as a diagnostic of the structure of the upper atmosphere, where it is formed. However, this is a very narrow feature, and therefore its measured value depends strongly on different broadening factors, like the rotation velocity of the star, turbulent velocity fields, and even the spectral resolution of the observations. In Table 1 we list the computed central intensities convolved with a spectral resolution of $0.15 \AA$, which corresponds with the observations used in Paper III.

In Figure 5 we show the profile obtained for the $\mathrm{K}$ line for several models of our hot series. It can be seen that, for these models, only a strong central emission is present and that there are no absorption wings. Therefore, the K1 minimum cannot be used as an indicator of the $T_{\min }$ structure for

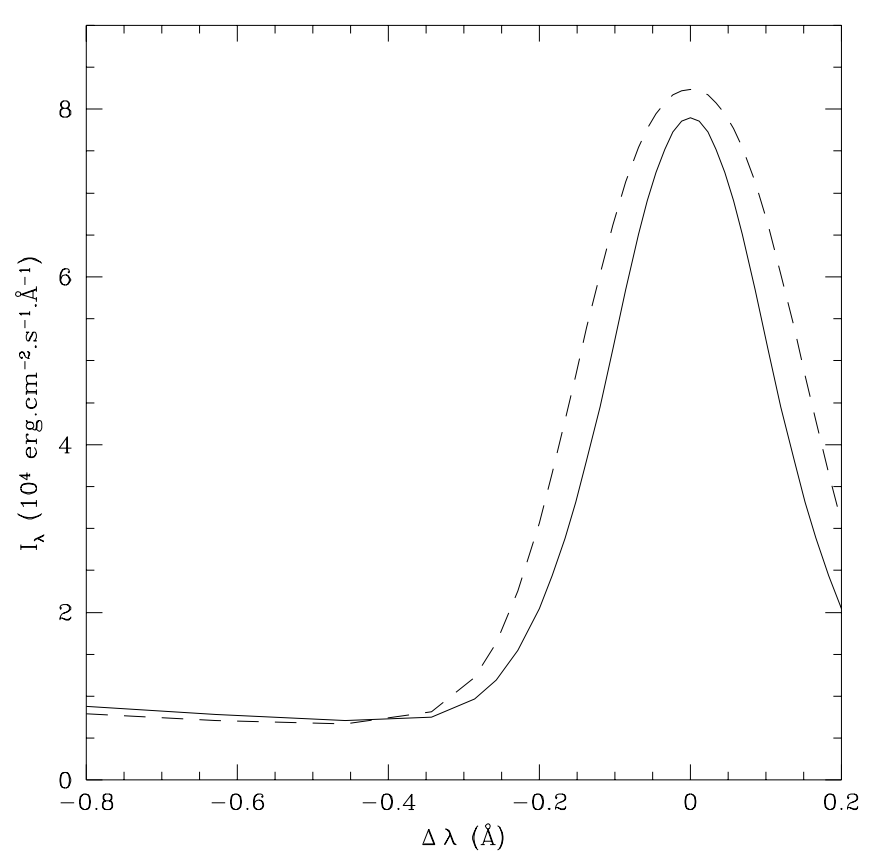

FIG. 4.- A detail of the $\mathrm{Ca}$ II $\mathrm{K}$ line profile around K1. Solid line: model $\mathrm{CM}+$; dashed line: model $\mathrm{CR}-$.

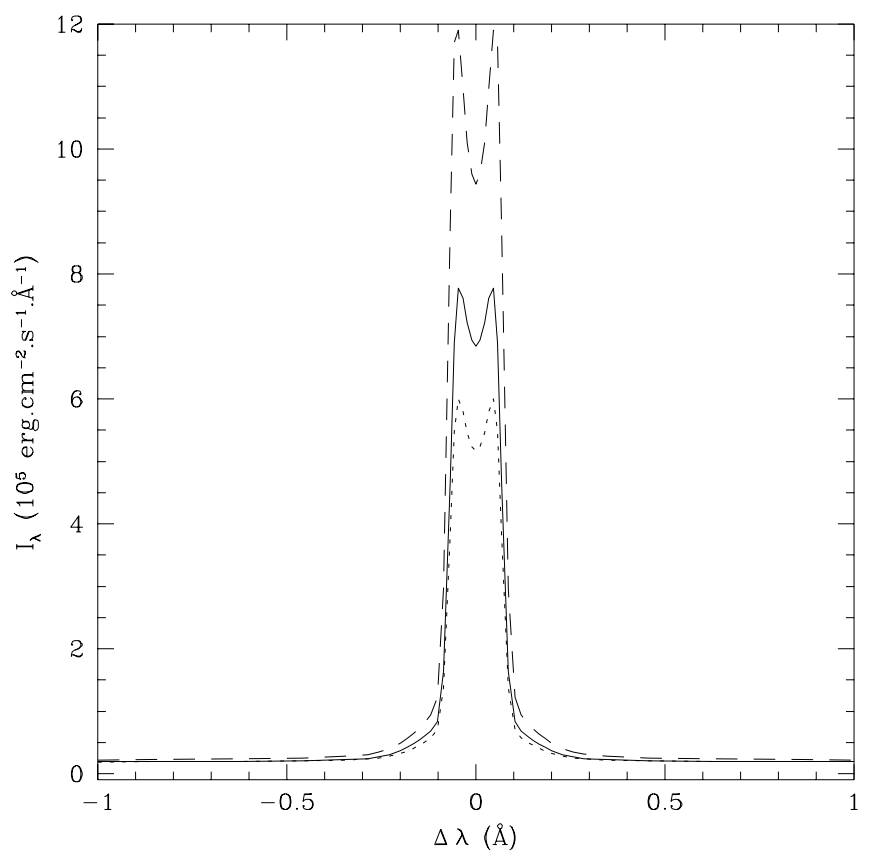

Fig. 5.-Profile of the $\mathrm{Ca}$ II $\mathrm{K}$ line for three models of our hot series. Solid line: model H; dashed line: model HS +; dotted line: model HR +.

$\mathrm{dMe}$ stars of this kind. We will see in the next section that a similar function can be performed by the $\mathrm{Na} \mathrm{D}$ lines.

\subsection{The $\mathrm{Na} \mathrm{D}$ Lines}

In Figure 6 we show the profiles of the central part of the $\mathrm{Na}$ D2 line for the same three models of the cold series shown in Figure 2. It can be seen that the profiles are different, particularly in the height of the line shoulder. These profiles are very similar to the ones given by Andretta et al. (1997) for their less active models.

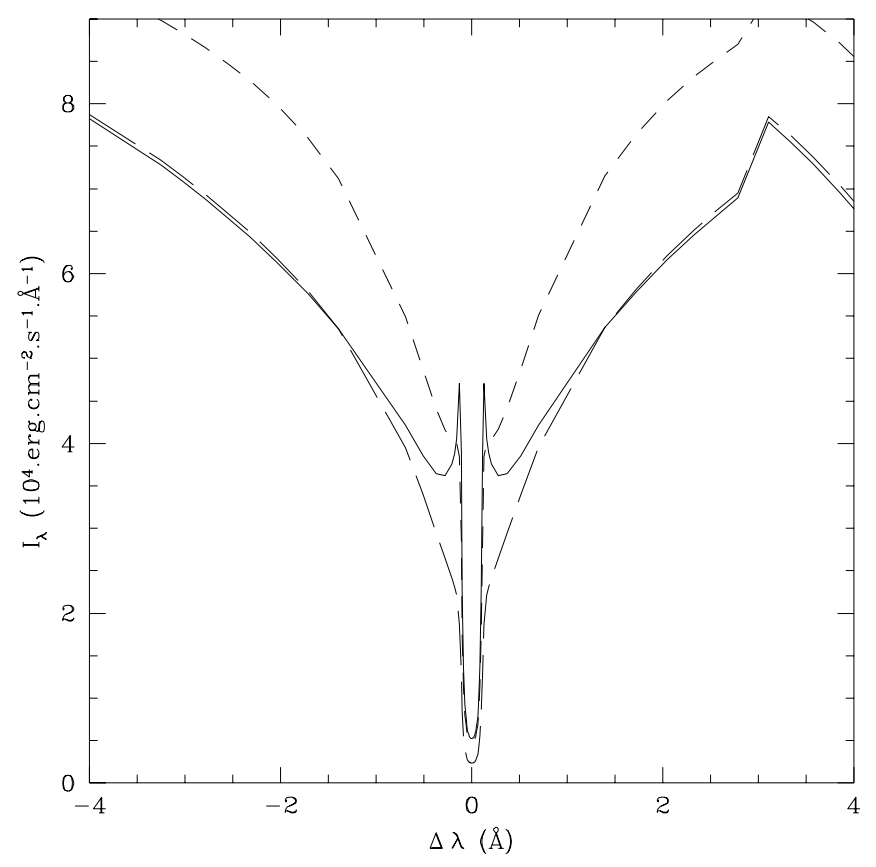

FIG. 6.-Profile of the Na D2 line for three models of our cold series. Solid line: model C; short-dashed line: model $\mathrm{CM}++$; long-dashed line: model CR + . 
However, this is not a spectral feature easy to use as an atmospheric diagnostic, since it is very sensitive to instrumental broadening, and high spectral resolution is needed. On the other hand, since the Doppler width of these lines is quite small, the line profiles in the core can be broadened by any velocity fields that could be present in the stellar atmosphere. For this reason, we did not use this line as a diagnostic tool for our cold model, and we use it only to check the final model.

On the other hand, for the hotter models the center of the $\mathrm{D}$ lines turns into emission, and therefore there is a minimum between the absorption wings and the emission center, a feature similar to the $\mathrm{K} 1$ minimum of the $\mathrm{Ca}$ II $\mathrm{K}$ line. We will note this feature as the $\mathrm{D}_{\min }$ from now on. In Figure 7 we compare the D2 profiles for three models of the hot series.

The intensity of the $D_{\min }$ is shown in column 7 of Table 2 for all models of our hot series. We see that the behavior of this feature is similar to the one for the K1 minimum, since it depends on the minimum temperature $T_{\min }$ and on the position of the first chromospheric rise $z_{4400}$ but remains unchanged when the temperatures of the high and midchromosphere are changed. On the other hand, the $\mathrm{D}_{\min }$ is less sensitive than the $\mathrm{K} 1$, since variations of the $T_{\min }$ of 40 $K$ give relative variations of the $D_{\min }$ of only around $10 \%$ $15 \%$, compared with a variation of around $20 \%$ for $\mathrm{K} 1$.

Therefore, the $\mathrm{D}_{\min }$ can be used as an atmospheric diagnostic for dMe stars in the same way that the K1 minimum can be used for the dM stars, since the structure of the $T_{\text {min }}$ region can be adjusted first to match the $D_{\min }$ intensity and the other features can be matched in a second step, adjusting the chromosphere. Also in this case, most $\mathrm{Na}$ is neutral, and therefore this line is insensitive to the details of the ionization balance.

Furthermore, the Na D line is not affected by partial redistribution, whereas the $\mathrm{Ca}$ II $\mathrm{K}$ line is so affected, and

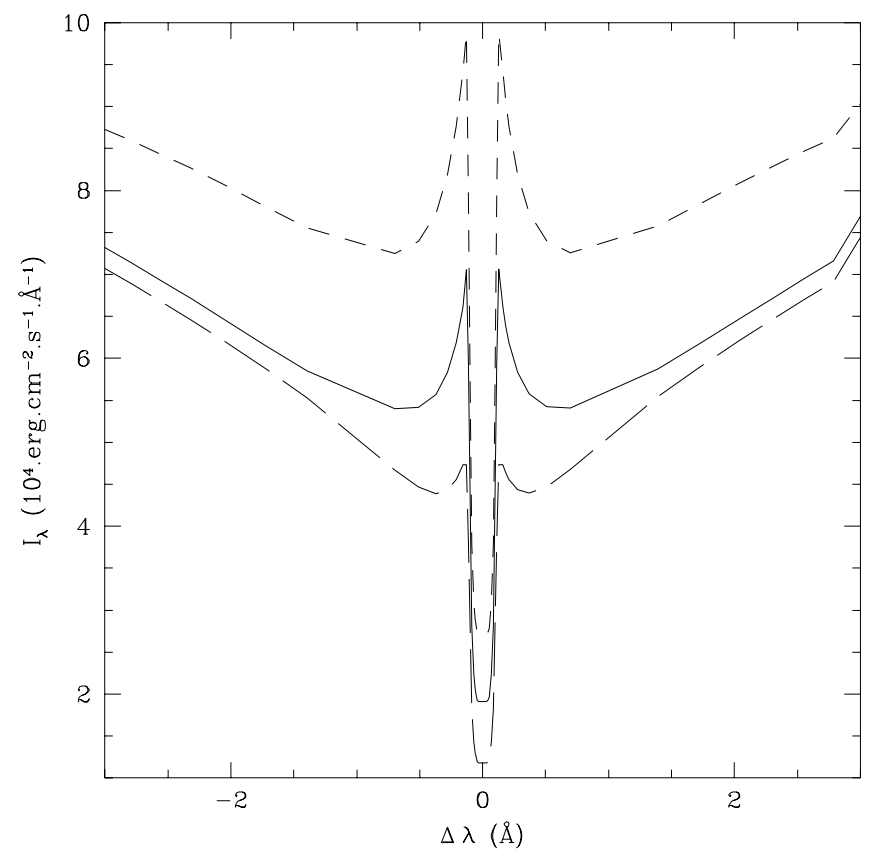

Fig. 7.-Profile of the Na D2 line for three models of our hot series. Solid line: model $\mathrm{H}$; short-dashed line: model $\mathrm{HM}+$; long-dashed line: model HR +. therefore the calculation of the $\mathrm{Na} \mathrm{D}$ line is simpler. Moreover, since for the cold stars the blue part of the spectrum is very faint, the integration times required to obtain profiles with a high signal-to-noise ratio are much greater for the $\mathrm{Ca}$ II lines than for the $\mathrm{D}$ lines.

\subsection{The Balmer Lines}

In Table 1 we show the central intensities of $\mathrm{H} \alpha$ and $\mathrm{H} \gamma$ for our cold models. We also list the fluxes for these two lines, with the continuum subtracted. As expected, the Balmer lines change with every modification of the base models.

In Figure 8 we show the $\mathrm{H} \alpha$ and $\mathrm{H} \gamma$ profiles for the same three models considered in Figures 2, 3, and 6 (models C, $\mathrm{CR}+$, and $\mathrm{CM}++$ ). These lines are very sensitive to

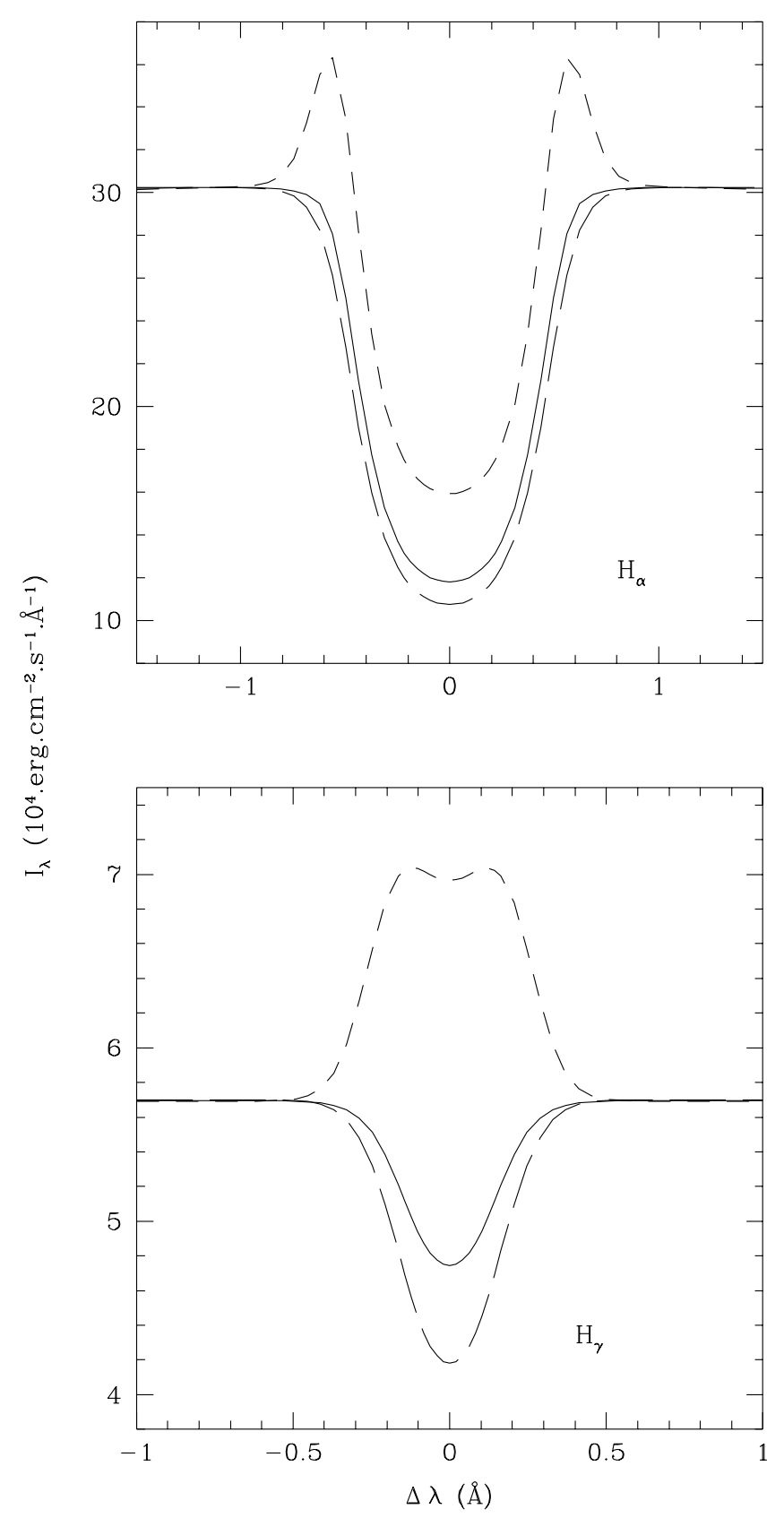

Fig. 8. - Computed profiles for $\mathrm{H} \alpha$ and $\mathrm{H} \gamma$ for three models of our cold series. Solid lines: model $\mathrm{C}$; short-dashed lines: model $\mathrm{CM}++$; longdashed lines: model $\mathrm{CR}+$. 
changes in the atmospheric model, since an increment of $100 \mathrm{~K}$ in the temperature minimum drives the $\mathrm{H} \gamma$ profile from absorption to emission and produces emission in the wings of $H \alpha$. However, it can be seen in the table that the changes in the line fluxes are much larger than the ones in the central intensities. This is particularly true for $\mathrm{H} \gamma$, for which, for example, going from model C to model CR + implies a reduction of $9 \%$ in the central intensity and an increment of $60 \%$ in the (negative) line flux.

This is due to the fact that $\mathrm{H} \gamma$ is a rather weak line, and therefore small changes in its absolute intensity result in large changes in its residual intensity. In fact, the absolute flux, integrated over $0.8 \AA$ (the approximate line width, as can be seen in Fig. 8), varies from 4.35 to $4.16 \mathrm{ergs} \mathrm{cm}^{-2} \mathrm{~s}^{-1}$ when the model is changed from model $\mathrm{C}$ to model CR + .

Therefore, the residual flux is not a reliable quantity to consider when comparing the models with the observations, since it is very unstable with respect to small changes in the model. This is particularly true for weak lines, and it is aggravated when the continuum level cannot be determined accurately, since a small variation in its determination produces changes in the residual flux much larger than any changes in the chromospheric structure.

The same problem arises with other quantities computed from the residual fluxes, which are usually considered as atmospheric diagnostics. For example, in Table 1 we also show the equivalent widths of $\mathrm{H} \alpha$ and $\mathrm{H} \gamma$, which are essentially the residual fluxes divided by the continuum intensity near the line. It can be seen that also in this case the variations are much larger than those in the central intensity. Another quantity usually considered is the Balmer decrement, which is the ratio of the residual fluxes and is therefore susceptible to the same problems.

An interesting fact to notice is that the ratio between the central intensities of the Balmer lines is insensitive to changes in the model. As an example, we also show in Table 1 the ratio of the central intensities of $\mathrm{H} \alpha$ and $\mathrm{H} \gamma$. It can be seen that it is possible to increase the $\mathrm{H} \alpha$ central intensity by $50 \%$ without affecting the line ratio.

This is due to the fact that the 5-3 line, and in fact all the Paschen lines, are formed just below $T_{\min }$, and the photons coming from the high chromosphere are mainly scattered photons. Therefore, in the region where the Balmer lines are formed, the source functions for the Paschen lines depend mainly on $\bar{J}$, which is the same for all the models of each series since the region below the temperature minimum is not changed. Since the source function for the Paschen lines is the same for all our models, the ratio between the number densities for the upper levels $(n \geq 3)$ is constant, and therefore the ratio of the Balmer source functions and intensities has to remain unchanged.

Therefore, only one of the Balmer lines can be used as a diagnostic of the chromospheric structure, and any variations in the ratio of the central intensities should be considered as a diagnostic of the region just below the temperature minimum.

On the other hand, it can be seen in Table 1 that there are different ways to obtain the same intensity of the Balmer lines. Models CC - and $\mathrm{CR}+$, for example, have the same central intensities for $\mathrm{H} \alpha$ and $\mathrm{H} \gamma$ (and, in fact, the same line profiles). This implies that different models can be built to explain the observations (for example, moving out the first chromospheric rise and compensating by increasing the chromospheric temperature). Therefore, the chromospheric structure cannot be constrained by fitting the Balmer lines alone, and some different diagnostic, such as the $\mathrm{Ca}$ II $\mathrm{K}$ lines, should be used.

However, there is still some indetermination which cannot be solved, at least with this set of observables. In fact, models $\mathrm{CC}+$ and $\mathrm{CS}+$ have the same values of the observables, within the usual error of the observations. It means that heating all the chromosphere uniformly or increasing the chromospheric slope has the same effects, as far as the mean temperature remains the same.

In Figure 9 we show the $\mathrm{H} \alpha$ and $\mathrm{H} \gamma$ profiles for the same three models considered in Figure 7 (models H, HR +, and $\mathrm{HM}+$ ). It can be seen that, in this case, the central intensity of $\mathrm{H} \alpha$ does not change much, and it is the maximum inten-

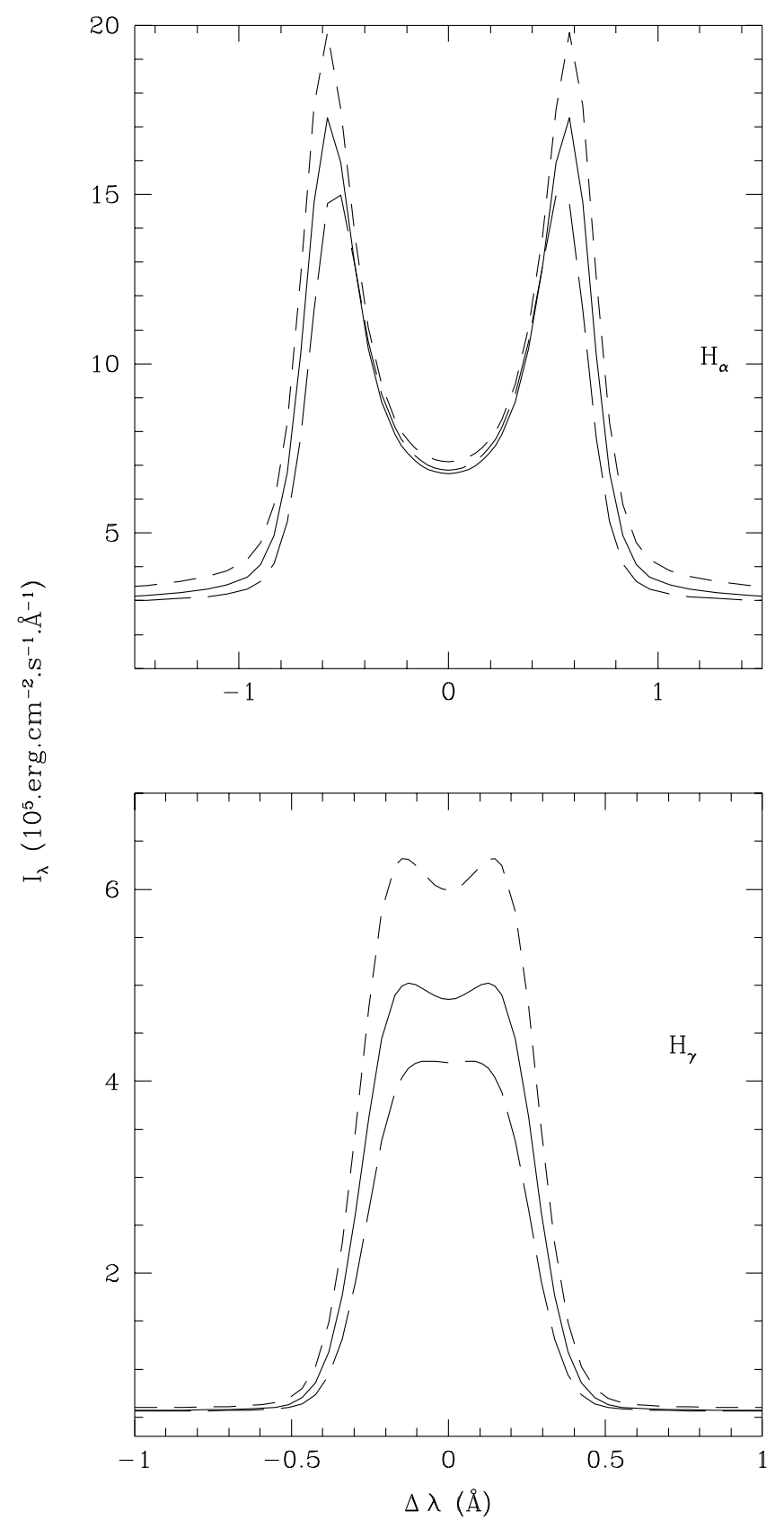

FIG. 9.-Computed profiles for $\mathrm{H} \alpha$ and $\mathrm{H} \gamma$ for three models of our hot series. Solid lines: model H; short-dashed lines: model $\mathrm{HM}+$; long-dashed lines: model $\mathrm{HR}+$. 
sity of this line which is relevant. Therefore, in Table 2 we list the maximum intensities for $\mathrm{H} \alpha$ and $\mathrm{H} \gamma$ for our hot models. In this case there are some variations in the line ratio, due to the fact that for these models the Paschen lines have a significant contribution from the chromosphere.

Another important fact to be noted from the values listed in Table 2 is that the Balmer lines are only slightly affected by changes in the structure of the upper chromosphere. The $\mathrm{H} \alpha$-line peak changes only slightly for models $\mathrm{HC}+$ and $\mathrm{HC}-$, where the temperature of the chromosphere is varied $250 \mathrm{~K}$ from the base model, and not at all for models HS + and HS - , where the temperature of the high chromosphere is changed by $600 \mathrm{~K}$. This implies that another feature is needed in order to better determine the temperature of this region.

In Table 2 we also list the integrated flux of the $\mathrm{Mg}$ II $k$ line. It can be seen that this flux in fact depends on the chromospheric temperature and can therefore help to constrain it. These fluxes were computed in PRD, since the CRD assumption for these lines is not valid.

However, also here models $\mathrm{HC}+$ and $\mathrm{HS}+$ have the same flux, and therefore for these models we still have the same indetermination between models heated uniformly or with different slopes that we found for the cold models.

\subsection{The Ly $\alpha$ Flux}

Another feature usually considered to constrain the atmospheric models is the Ly $\alpha$ flux. Houdebine \& Doyle (1994a) found an observed value for the Ly $\alpha$-to- $\mathrm{H} \alpha$ flux ratio of 1 for AU Mic and used it to constrain the form of the transition region. Houdebine, Doyle, \& Kościelecki (1995) derived values for this ratio ranging from 1.4 to 21 . Doyle et al. (1997) used computed Ly $\alpha$ profiles to estimate attenuation factors for this line and found that the intrinsic ratio for the active dMe stars ranges from 3 to 5 . However, all these works computed the Ly $\alpha$ line assuming complete redistribution, which, as we pointed out in Paper IV, can introduce errors of as little as $10 \%$ for the hot models or as much as a factor of 5 for the cold models.

In the tables we list the Ly $\alpha$ flux for each model and the ratio of the $L y \alpha$ to the $H \alpha$ fluxes. In Table 2 we can see that, for the active stars, this ratio remains between 1 and 2 for all the models. It can also be noted in the tables that moving downward the position of the transition region, as given by the models labeled $\mathrm{Z}-$, enhances the flux ratio. For the cold models, moving the transition region changes the Ly $\alpha$ flux without altering the other observable features.
Therefore, the Ly $\alpha$ flux can be used to determine the position of the transition region, once the rest of the atmosphere has been constrained. It can also be noted that moving the transition region in or out by only $40 \mathrm{~km}$ for the cold models, or $100 \mathrm{~km}$ for the hot ones, implies changes in the flux, or in the flux ratio, larger than $10 \%$. To obtain a $30 \%$ decrement of the Ly $\alpha$ flux it is necessary to move the transition region outward by $200 \mathrm{~km}$ for the cold model and $300 \mathrm{~km}$ for the hot one.

\section{CONCLUSIONS}

In this paper we investigate how different modifications in the temperature versus $z$ distribution assumed for a given $\mathrm{dM}$ or dMe star modify the chromospheric spectral features. In this way we study which information on the different regions of the stellar atmosphere is provided by several lines usually observed, and therefore which observations are necessary to assure that the model inferred is unique.

We found that, for the models representing $\mathrm{dM}$ stars without emission in the Balmer lines, the $\mathrm{K} 1$ minimum of the $\mathrm{Ca}$ II $\mathrm{K}$ line is a good indicator of the structure of the temperature-minimum region, and does not vary when the chromosphere is modified. On the other hand, for the dMe stars which have the Balmer lines in emission, the $\mathrm{K}$ line turns into emission completely, and the $\mathrm{K} 1$ minimum is no longer visible. Therefore, for these stars the $\mathrm{K}$ line does not provide useful information and can be omitted from the observations.

However, for these hot models the Na D lines show a central emission, and the minimum which appears can be used to determine the position and temperature of $T_{\min }$ in much the same way as K1 for the cold models.

Once the $T_{\min }$ is constrained using either the $\mathrm{Ca}$ or the $\mathrm{Na}$ lines, the Balmer lines can be used to determine the temperature and slope of the chromosphere. For the cold models, the information provided by the different Balmer lines is redundant, since the ratio of the central intensities remains constant.

For the hot models, the Balmer lines do not help to determine the temperature of the highest layers of the chromosphere, and another spectral feature, for example the $\mathrm{Mg}$ II $k$ flux, is needed. However, for both series of models, it is possible to determine only a main value of the chromosphere, and not its exact slope.

Finally, the position of the transition region can be determined if the $L y \alpha$ flux is available.

\section{REFERENCES}

Andretta, V., Doyle, J. G., \& Byrne, P. B. 1997, A\&A, 322, 266

Avrett, E. H. 1985, in Chromospheric Diagnostics and Modeling, ed. B. W. Lites (Sunspot: NSO), 67

Ayres, T. R., \& Linsky, J. L. 1976, ApJ, 205, 874

Cram, L. E., \& Mullan, D. J. 1979, ApJ, 234, 579

Doyle, J. G., Mathioudakis, M., Andretta, V., Short, C. I., Jelinski, P. 1997, A\&A, 318, 835

Falchi, A., \& Mauas, P. J. 1998, A\&A, 336, 281 (Paper IV)

Giampapa, M. S., Worden, S. P., \& Linsky, J. L. 1982, ApJ, 258, 740

Houdebine, E. R., \& Doyle, J. G. 1994a, A\&A, 289, 169
Houdebine, E. R., \& Doyle, J. G. 1994b, A\&A, 289, 185

Houdebine, E. R., Doyle, J. G., \& Kościelecki, M. 1995, A\&A, 294, 773

Kurucz, R. L. 1991, in Precision Photometry: Astrophysics of the Galaxy, ed. A. G. D. Philip, A. R. Upgren, \& K. A. Janes (Schenectady: L. Davis) Mauas, P. J., \& Falchi, A. 1994, A\&A, 281, 129 (Paper I)

. 1996, A\&A, 310, 245 (Paper II)

Mauas, P. J., Falchi, A., Pasquini, L., \& Pallavicini, R. 1997, A\&A, 326, 249 (Paper III)

Thatcher, J. D., Robinson, R. D., \& Rees, D. E. 1991, MNRAS, 250, 14 